\title{
Les politiques linguistiques de la France et le statut des langues en Belgique
}

Guy Jucquois

\section{OpenEdition}

1 Journals

Édition électronique

URL : http://journals.openedition.org/ries/4226

DOI : $10.4000 /$ ries.4226

ISSN : 2261-4265

Éditeur

Centre international d'études pédagogiques

\section{Édition imprimée}

Date de publication : 1 septembre 1994

Pagination : 47-61

ISSN : 1254-4590

\section{Référence électronique}

Guy Jucquois, "Les politiques linguistiques de la France et le statut des langues en Belgique », Revue internationale d'éducation de Sèvres [En ligne], 03 | 1994, mis en ligne le 17 avril 2015, consulté le 10 décembre 2020. URL : http://journals.openedition.org/ries/4226 ; DOI : https://doi.org/10.4000/ries. 4226

Ce document a été généré automatiquement le 10 décembre 2020.

(c) Tous droits réservés 


\title{
Les politiques linguistiques de la France et le statut des langues en Belgique
}

\author{
Guy Jucquois
}

\section{Introduction}

1 L'étude des répercussions en Belgique, et selon les communautés linguistiques, de la politique linguistique de la France, particulièrement dans le cadre de la construction de l'Europe, soulève une double question d'information et de méthode.

2 L'interprétation en Belgique des positions françaises suppose de reformuler ces positions selon nos perceptions, wallonne et flamande, et d'expliciter nos réactions par rapport aux politiques françaises. Mais comment apprécier ces perceptions? Dans l'impossibilité d'effectuer un sondage ou, à défaut, de recourir à des études préalables - qui n'existent pas, à notre connaissance-, il a donc fallu nous résoudre à travailler selon nos informations et nos impressions personnelles, vérifiées et rectifiées éventuellement d'après des conversations avec des personnes « averties » appartenant aux communautés néerlandophone et francophone de Belgique ${ }^{1}$.

Une autre difficulté provenait du caractère contesté de la réalité belge, c'est-à-dire de l'existence de traits culturels et d'une sensibilité que les Belges auraient en commun et qui les distingueraient respectivement des autres néerlandophones et des autres francophones. Si certains prétendent qu'il n'existe pas de Belgique, d'autres affirment, par contre, la réalité de la « belgitude ». Les querelles belges amusent l'étranger ou, dans le meilleur des cas, le rendent perplexe. Nos réalités culturelles sont souvent mal connues, même chez nos proches voisins dont l'ignorance à notre égard nous stupéfie souvent. Les faibles dimensions du pays, en contraste d'ailleurs avec son importance historique et contemporaine, nous recommandaient cependant d'assumer cette méconnaissance même si elle s'accompagnait souvent d'une ironie gentiment moqueuse. La multiplication des relations et l'unification européenne imposent aujourd'hui à tous de mieux se connaître. 


\section{La situation de la Belgique}

\section{Une situation historiquement inégalitaire}

4 La Belgique contemporaine fut créée par la révolution de 1830 qui provoqua la séparation de notre pays et de la Hollande, situation qui fut reconnue en droit international par la Conférence de Londres (novembre-décembre 1830), suivie un mois plus tard d'un protocole qui amputait le pays de la Flandre zélandaise, d'une partie du Limbourg, qui devint le Limbourg hollandais, et d'une partie du Luxembourg.

Quoiqu'occupée durant de nombreux siècles par diverses puissances étrangères ou, au contraire, morcelée et répartie entre l'Empire germanique et la France ou encore indépendante et forte, bien que composite, comme sous les ducs de Bourgogne, la Belgique donna naissance aux Mérovingiens, aux Carolingiens et c'est dans nos terres que naquit également Charles-Quint. Le lecteur ne verra dans ce rappel que la confirmation d'une originalité historique qui réside essentiellement dans de constants compromis et métissages entre les composantes germaniques et romanes et qu'illustrent aussi bien les querelles de ménage que le caractère proprement interminable du divorce belge.

6 L'occupation hollandaise, simple épisode (1814-1830) dans notre longue histoire, se termina par la création d'un État-tampon dont les limites ne correspondaient pas à l'aire culturelle qui s'était progressivement développée depuis l'Antiquité et qui se trouva ainsi amputée de ses prolongements. Durant ces quinze ans, la domination hollandaise avait cependant détérioré le climat des relations entre les communautés néerlandophone et francophone de Belgique : la volonté d'imposer le néerlandais avait créé des réactions en faveur du français qui, de ce fait, s'affirma également dans la bourgeoisie flamande. La francisation des élites flamandes, déjà entreprise avant la période hollandaise, fut d'autant plus mal ressentie par les masses flamandes qu'elle redoublait de grosses difficultés économiques et sociales spécifiques au Nord du pays.

7 La langue française devint ainsi, dans les régions belges de langue néerlandaise, signe de la domination politique, sociale, économique et culturelle exercée sur les néerlandophones qui ne reniaient pas leur identité culturelle. Cette situation, différente et perçue différemment selon les régions, devait donner lieu à une longue incompréhension entre les deux principales communautés linguistiques belges. Au sud, en effet, bien que, depuis des siècles, le français ait progressivement supplanté trois autres dialectes d'oï - le wallon, le picard et le lorrain - et que, là également, la langue française fût signe de domination, son usage apparut cependant comme moins marqué qu'au nord. La longue lutte d'indépendance des Flamands fut donc largement incomprise au sud du pays et les succès que ces derniers remportèrent peu à peu sur tous les plans exacerbèrent encore l'amertume et la rancœur de beaucoup de Bruxellois francophones et de Wallons, victimes du déclin de leurs régions.

\section{Langues, régions et communautés}

$8 \quad$ Aujourd'hui, au terme provisoire ${ }^{2}$ d'une longue évolution et de nombreux compromis qui marquèrent la fin de multiples conflits entre les communautés culturelles belges ${ }^{3}$, le pays a acquis une structure fédérée. Cette structure fut le résultat des dernières négociations communautaires, mais elle porte également la marque des étapes antérieures et, 
particulièrement, les éléments de contradictions issus d'une tension fort ancienne entre ceux qui souhaitaient la fédéralisation de la Belgique et ceux qui s'y opposaient ${ }^{4}$. Il importe, en effet, de bien comprendre ces éléments pour apprécier comment les politiques linguistiques de la France sont perçues différemment selon les situations.

9 L'évolution du pays depuis son indépendance avait abouti à donner progressivement au néerlandais les mêmes droits qu'au français. Cette lutte s'acheva par l'adoption d'une nouvelle série de lois linguistiques votées de 1962 à 1966 et par la révision constitutionnelle de 1971 qui fixait la frontière linguistique, supprimait logiquement le volet linguistique du recensement et consacrait le principe de territorialité pour l'ensemble du territoire sauf dans ce qui deviendra la région de Bruxelles-Capitale où s'appliquerait le principe de personnalité. Cette phase de la réforme de l'État est connue sous le nom de communautarisation puisque, pour la première fois, y était reconnue l'existence de deux communautés linguistiques, néerlandaise et française ${ }^{5}$.

L'étape suivante de la réforme s'appelle la phase de régionalisation et date des années 1980. Trois régions furent instituées. La région flamande se confondit - et ceci est important avec la communauté flamande et fut gérée par le Conseil flamand qui décida de fixer sa capitale à Bruxelles dont les citoyens flamands dépendraient des mêmes institutions. Cette confusion entre des entités (communautés et régions) voulues essentiellement par des unitaristes principalement francophones et visant à freiner la fédéralisation du pays permit aux néerlandophones, déjà soudés par une longue lutte commune, de présenter sur la plupart des questions identitaires un front uni. Leur évolution rendait donc les néerlandophones belges particulièrement aptes à aborder la troisième phase des réformes de l'État, la fédéralisation qui débuta en 1988 et qui accroissait encore les compétences accordées aux régions et communautés ${ }^{6}$.

11 Il n'en alla pas de même du côté des francophones. Issus des diverses régions du pays, ayant des sensibilités et des intérêts souvent divergents sinon contradictoires ${ }^{7}$, leurs hésitations s'ajoutèrent aux réticences flamandes concernant l'autonomie à accorder à la région bruxelloise ${ }^{8}$. Répartis, comme on le précisera plus bas, entre quatre groupes d'importance inégale, les francophones belges dans leur majorité demeurèrent longtemps unitaristes. Deux raisons principales y concouraient: appartenant historiquement au groupe dominant, beaucoup de francophones belges ne voyaient pas de motif de modifier une situation qui leur paraissait «normale » et «naturelle», position injuste, mais qui, avec le renversement des rapports de force dans la société belge, devint une position absurde. Par ailleurs, les intérêts autres que linguistiques et, partiellement, culturels des quatre composantes des francophones belges les dressaient les uns contre les autres, tandis que le recours à la même langue - le français - maintenait entre eux un ciment d'unité. L'ambiguïté des positions unitaristes permettait d'occulter ces divergences, elle affaiblissait cependant d'autant la position de la communauté française de Belgique.

Avec le temps, sans pour autant renoncer à la défense du français langue étrangère en Flandre et sans négliger le sort des francophones de la périphérie bruxelloise ${ }^{10}$, deux groupes importants ont émergé, groupes reconnus d'ailleurs par les réformes constitutionnelles, les francophones de la région de Bruxelles-Capitale et ceux de la région wallonne. 


\section{Les sensibilités contemporaines}

13 Les luttes dont on faisait état ci-dessus s'achèvent aujourd'hui. La Belgique est devenue fédérale, sans qu'on puisse affirmer pour autant que les passions et les revendications y soient toutes satisfaites, ni même exprimées. Du passé, les néerlandophones ont conservé le souvenir de luttes contre un pouvoir francophone, local (les «fransquillons" ou bourgeoisie francophone flamande, ou national, qui les opprimait, le souvenir également d'une infériorité économique et culturelle. Le présent fait contraste par son dynamisme et la réussite actuelle est associée chez beaucoup à la prise de conscience des valeurs identitaires. Le Flamand est d'abord flamand, accessoirement belge, souvent européen, jaloux de son autonomie parce que fier de son identité.

14 La situation des francophones est plus complexe. Ils se répartissent en quatre groupes d'inégale importance ${ }^{11}$ : en région flamande subsiste une bourgeoisie francophone, le plus souvent bilingue d'ailleurs, dont l'importance numérique est faible; la région de Bruxelles-Capitale est francophone à environ 75 à $85 \%$ selon les estimations ${ }^{12}$; en région flamande encore, mais dans la périphérie bruxelloise, vit une population francophone immigrée qui atteindrait, selon les estimations francophones, de 4 à 500000 habitants ; la région wallonne enfin est entièrement francophone ${ }^{13}$ à l'exception d'une communauté germanophone, reconnue et protégée par la loi, localisée à l'est du pays et forte d'environ 67000 personnes $^{14}$. Chacun de ces sous-groupes développe envers la communauté flamande une attitude propre: généralement discrète, la bourgeoisie francophone minoritaire des Flandres adhère aux objectifs de la population flamande dont elle partage la sensibilité tout en maintenant, dans la tradition familiale, son attachement à la culture française des élites. La population francophone immigrée de la périphérie bruxelloise provient pour l'essentiel de Bruxelles même ou de Wallonie. Sa sensibilité n'est différente de celle de ses congénères demeurés en ces lieux que par la tension, plus ou moins grande selon les moments, mais toujours significative entre ces francophones et une large frange de la communauté néerlandophone. Les francophones de la périphérie bénéficient, dans six communes, du régime dit de «facilités » qui permet à chaque citoyen de s'adresser dans sa langue à l'administration communale et de recevoir l'enseignement de base dans la langue maternelle, en l'occurrence en français ${ }^{15}$. Ce régime particulier a été reconnu dans les diverses révisions constitutionnelles. Il soulève néanmoins la polémique, car, du côté néerlandophone, on craint qu'il ne soit étendu à d'autres communes actuellement officiellement flamandes ou qu'on ne passe progressivement d'un régime à «facilité » à un régime de bilinguisme légal. Du côté francophone, par contre, on évoque les difficultés et la précarité du régime actuel, soumis, dit-on, à la « bonne volonté » flamande et on ne manque pas de faire remarquer le caractère paradoxal de la situation où une majorité estimée de francophones est traitée légalement comme une minorité plus ou moins protégée. Bien que se sentant moins directement menacés par les néerlandophones, les Bruxellois francophones ressentent mal ce qu'ils considèrent comme des manifestations de la volonté de «flamandisation » de la capitale ${ }^{16}$, tels les affichages administratifs et commerciaux répartis généralement par moitié, telle encore la répartition du personnel dans les administrations communales ou fédérales d'après le régime linguistique, telle aussi la presse et la propagande flamandes dont certaines expressions sont très agressives envers les francophones bruxellois. 
Les Wallons, enfin, qui, bien que constituant la grosse majorité des francophones belges ${ }^{17}$, n'ont acquis qu'imparfaitement et très tardivement le sentiment de leur appartenance à une communauté francophone. Dans la mesure où ce dernier émerge et se fortifie, il semblerait qu'il se confonde chez nombre d'entre eux avec le sentiment de leur appartenance à la région wallonne. En d'autres termes, beaucoup de Wallons se sentent francophones parce que Wallons, tandis que chez de nombreux Bruxellois francophones, leur identité francophone passerait avant leur identité bruxelloise. Cette différence de sensibilité a comme conséquence une perception différente des identités régionales et francophone: pour les uns, l'appartenance à la francophonie l'emporte sur l'appartenance régionale, pour les autres, ce serait l'inverse. D'où également des tensions sur les plans politique et institutionnel selon que la communauté française de Belgique l'emporte, dans l'esprit des citoyens, sur la région de Bruxelles-Capitale et sur la région wallonne ou inversement.

\section{Les politiques linguistiques de la France}

\section{Des choix politiques multiples}

La France semble, vue de la Belgique, osciller entre plusieurs politiques linguistiques différentes, parfois complémentaires, éventuellement contradictoires. Ce dernier aspect s'atténue cependant lorsqu'on interprète les politiques linguistiques françaises en liaison avec des couples tensoriels qui en rendent mieux compte : les choix apparaissent alors comme des tentatives de compromis que partagent sans doute, dans ce domaine, d'autres pays européens, quoique, chez ces derniers, les oppositions semblent, pour des raisons historiques et conjoncturelles, moins tranchées que dans le cas de la France.

Trois couples tensoriels sous-tendent apparemment les choix politiques linguistiques de la France : l'opposition entre la rétrospection et la prospective, l'opposition entre la politique intérieure et la politique extérieure, l'opposition entre des valeurs d'uniformité et des valeurs de diversité18. Si le jeu politique français est l'objet de tensions entre les diverses valeurs que représentent ces trois couples oppositionnels, c'est non seulement en fonction du regard multiple et nuancé que les Français portent sur eux-mêmes et sur le monde, mais aussi en réponse à la variation des regards supposés du monde sur la France. Si ces éléments interviennent également dans l'élaboration de la politique de n'importe quel État, dans le cas de la France, le contraste entre ces valeurs est, pour ainsi dire, exacerbé et cela d'autant plus que ce pays ambitionne de remplir une fonction internationale importante et que nombre de contemporains lui créditent un rôle dont le caractère davantage «moral » compense peut-être la perte d'influence sur le plan plus primaire de la « force».

18 Quoi qu'il en soit, en matière de langues, comme dans d'autres domaines, la politique française n'apparaît pas cohérente et constante, mais soumise à diverses forces dont il importe de montrer le jeu. L'opposition entre la rétrospection et la prospective se manifeste par une différence entre les nostalgiques de la grandeur passée de la France et ceux que préoccupe davantage l'adaptation à un monde en pleine mutation. Les premiers se soucient de défendre le français en tout lieu où son usage international pourrait être remis en cause, soit dans la défense de positions acquises et héritées du passé, soit en tentant d'imposer l'usage de notre langue en de nouveaux lieux internationaux. Les 
seconds ne se soucient plus guère d'un passé glorieux et sont assez enclins à passer au « tout à l'anglais » de ceux qu'ils estiment être les maîtres de l'avenir.

19 L'opposition entre la politique intérieure et la politique extérieure est d'autant plus grande en France que ce pays est tiraillé par d'autres tensions. En effet, le tiraillement, sinon la contradiction, qui existe entre l'attitude politique intérieure et l'attitude politique extérieure met en évidence combien peu les mêmes principes servent de ligne directrice dans le pays lui-même ou en dehors de ses frontières. Si, au nom de principes libertaires dont elle se ferait l'avocat, la France revendique, à l'extérieur, la liberté pour chaque communauté de défendre son identité et donc sa langue, comment pourrait-elle légitimer son attitude, souvent autoritaire sinon condescendante, envers ses propres minorités linguistiques? Si, au contraire, elle tente de justifier, en son sein, le manque de moyens et l'absence de reconnaissance de ses minorités ethniques, si, donc, elle parait se situer en un rapport de domination envers ces dernières, où pourrait-elle trouver les arguments pour prendre la tête d'une fronde des « dominés " ? L'impasse est cependant moins absolue qu'il n'y parait de prime abord, puisque de nombreux pays connaissent l'opposition entre la volonté d'une indépendance extérieure et l'autorité d'un contrôle intérieur et que, dès lors, des connivences permettent de maintenir la bonne conscience. La troisième opposition, entre des valeurs d'uniformité et des valeurs de diversité, se situe sur un plan plus général et renvoie à des modèles de société qui partagent la société française entre des valeurs de contrôle et d'obéissance ou des valeurs de liberté et d'indépendance. La France est le pays où se produisit une des grandes secousses, la Révolution de 1789, qui marquèrent l'avènement du monde contemporain: s'y élabora notamment le centralisme politique et administratif, mais s'y formula également la Déclaration des droits de l'homme. Ces éléments connotent fortement les politiques françaises en matière de langue, songeons aux positions prises sur ce plan par l'abbé Grégoire.

21 Enfin, le jeu de ces différentes forces fluctue avec le temps : les politiques françaises de la langue sont évolutives. D'abord, parce que les générations qui se succèdent ne ressentent pas toutes les questions de langue de la même manière. Ensuite, parce que, depuis quelques décennies, les rapports de force entre les grandes langues internationales se sont grandement modifiés : le prestige du français se rapproche de plus en plus de celui d'autres langues internationales telles que l'allemand, l'espagnol ou le russe et est, par contre, de moins en moins à égalité avec l'anglo-américain. Aussi, parce que les francophones participent aux revendications identitaires qui marquent notre époque au même titre que les membres de toutes les communautés culturelles et linguistiques de la terre. En dernier lieu, parce que des jeux d'alliance commencent à se dessiner dans ces questions et que le sort de ces alliances dépend pour une large part d'une appréciation correcte de l'ensemble des facteurs énumérés ci-dessus.

\section{Les politiques linguistiques françaises}

22 Il y a lieu de distinguer deux volets dans les politiques linguistiques françaises : quelles sont les attitudes des Français envers leur langue et, d'autre part, quelles sont leurs politiques envers l'usage des langues en France et ailleurs?

En comparaison avec d'autres communautés linguistiques, beaucoup de francophones développent envers leur langue une attitude obséquieuse d'hyper-correction et de rigorisme qui rappelle davantage les scrupules maniaques qu'une simple et affectueuse 
intimité avec ce qui constitue un des principaux éléments de l'identité communautaire. En l'espèce, deux corollaires en découlent: l'énorme difficulté à proposer des modifications de la langue qui aient une chance d'être acceptées ${ }^{19}$ et la préférence accordée par beaucoup à un abandon de notre langue dans certaines situations plutôt que d'accepter des concessions sur ce point. Tout ceci entraîne de funestes confusions chez nombre de francophones de France qui s'imaginent que la défense de leur identité passe par la défense de ce qu'ils pensent être la pureté de la langue. D'où le quiproquo que représentent, par exemple, les interviews accordées régulièrement par des linguistes de renom et qui ne portent que sur des questions de langue telles que les nombreux emprunts à l'anglais ou la perte de l'orthographe, alors que les enjeux concernent des questions économiques, sociales et politiques. À notre avis, il s'agit moins de se préoccuper des difficultés internes à la langue que des difficultés externes: l'importance de l'enjeu n'est pas l'accord d'un participe passé ou le maintien d'un accent circonflexe dans tel mot dans un contrat rédigé en français, mais bien simplement de savoir si, à l'avenir, des francophones seront encore autorisés à passer contrat et à travailler, chez eux et entre francophones, en français ${ }^{20}$.

Indépendamment de cette question, les politiques linguistiques françaises paraissent encore largement liées à la défense de positions historiques, sans doute largement dépassées ou en voie de l'être incessamment. Certes la défense du français, seule langue internationale face à l'anglais, peut encore être assurée, dans une certaine mesure, par quelques pays qui choisissent ainsi un moyen terme entre l'uniformisation qui ne les tente guère et le maintien problématique d'identités très diversifiées. Le français joue dans ce cas le rôle de défenseur de la veuve et de l'orphelin. Pour le reste, le français comme langue, officielle et de travail, dans les organismes internationaux, est au mieux sur la défensive et dans la plupart des cas en recul. Or, on le conçoit aisément, si les États membres des organismes internationaux acceptent de recourir à des langues médiatrices, c'est uniquement pour assurer la commodité des échanges dans les réunions internationales. Dès lors que les langues de travail sont des langues étrangères pour la plupart des participants, le choix de telle ou telle langue est à l'évidence lié à une tradition et plus précisément au fait qu'un enseignement de haut niveau dans cette langue ait été et soit assuré dans tel pays. Si les choix scolaires changent, il en découlera, avec un décalage prévisible dans le temps, une modification dans les usages et dans le choix des langues. Nous sommes actuellement dans cette période de transition en ce qui concerne l'étude respective $d u$ français et de l'anglais. La défense du français à côté du seul anglais appartient donc, selon nous, à la défense du passé.

Le recul du français dans les organisations internationales conduit parfois les autorités politiques de la France à une stratégie de rechange. La chute des régimes communistes en Europe de l'Est a multiplié, les cinq dernières années, les adhésions au Conseil de l'Europe. Les nouveaux membres recourant de préférence à l'anglais, la fréquence d'utilisation de cette langue a crû relativement à celle du français, précipitant au détriment de ce dernier un déséquilibre qui avait été amorcé déjà auparavant. Or, dans ce cas et par le jeu de la réciprocité, les francophones auraient pu trouver, notamment auprès des germanophones et des hispanophones, une alliance dans la mesure où ils auraient accepté et promu l'allemand et l'espagnol dans les mêmes instances. Par contre, dans le cas de l'Eurocorps ou dans celui des langues de travail de l'Union européenne, la politique linguistique française consiste à envisager des alliances avec d'autres communautés linguistiques $^{21}$. Cette politique, plus pragmatique, risque cependant de 
susciter au français d'autres « ennemis » parmi les communautés qui n'ont pas fait l'objet d'alliance : à cet égard, l'exemple de l'Eurocorps est significatif, le choix du français et de l'allemand comme langues de commandement excluait en fait le néerlandais, d'où les vexations et les protestations que l'on connaît.

Une dernière politique possible consisterait - pour des raisons éthiques ou politiques - à soutenir toutes les communautés qui tendent à obtenir une meilleure reconnaissance de leur langue. Il s'agirait d'appuyer les revendications de groupes qui, par hypothèse, seraient minoritaires, soit dans l'absolu, soit dans le contexte où ces revendications sont émises. Une semblable politique serait très habile de la part du groupe qui vise à la domination sur le plan mondial : en effet, elle apparaît comme généreuse et libératrice et entrave fortement l'action de groupes moins puissants en vue d'un éventuel partage du leadership. Concrètement, ce serait la politique linguistique que pourrait suivre la communauté anglo-américaine - elle serait, par contre, débilitante pour la communauté francophone.

La France semble donc devoir adopter une politique moyenne, orientation qu'apparemment elle n'a pas encore choisie énergiquement. Quelques indices montrent néanmoins qu'elle pourrait s'engager dans un avenir prochain dans cette direction. Renonçant à un leadership linguistique dont elle n'a pas les moyens, elle pratiquerait une politique d'alliances de manière à éviter l'uniformisation par le seul recours à l'angloaméricain $^{22}$. Insistons sur le fait que des alliances de ce genre doivent être précédées d'études globales, c'est-à-dire politique, économique, sociale, etc. et ne pas se limiter à de platoniques accords « culturels».

\section{La perception belge}

\section{Du côté des francophones}

Les francophones de Belgique, à quelque catégorie qu'ils appartiennent ${ }^{23}$, se sentent fortement concernés par les différents aspects des politiques françaises dans le domaine des langues en général et du français en particulier. Sans doute ${ }^{24}$, retrouverait-on chez les francophones belges les mêmes tendances que chez les francophones français. Toutefois la rencontre quotidienne, les luttes et les tensions, mais aussi les liens et les amitiés, avec des membres des autres communautés (néerlandophone et germanophone), le souvenir d'innombrables occupations étrangères et les nécessaires adaptations qu'elles ont exigées, la longue tradition enfin d'une étude des langues étrangères (plus dynamique il est vrai au nord qu'au sud du pays), la présence aujourd'hui de forts contingents d'étrangers sur le sol belge, tout cela conduit les francophones belges à davantage de réalisme et de tolérance qu'ils n'en perçoivent chez leurs voisins français.

Quoique réputés pour avoir suscité et formé les meilleurs spécialistes dans le domaine de la grammaire française ${ }^{25}$ et bien qu'ayant largement contribué au renom des lettres françaises, les francophones belges sont, dans l'ensemble, beaucoup moins attachés au respect scrupuleux de normes langagières. On fut surpris en Belgique par les réactions violentes que provoqua, en France, la très modeste réforme orthographique à laquelle avait contribué également le grammairien belge André Goosse. D'ailleurs, l'usage fréquent de belgicismes, dont nombre se justifient, et l'habitude d'entendre notre langue être quelque peu malmenée par de méritants allophones qui nous font l'honneur de la parler nous a rendus plus conciliants envers la correction de la langue. Les réactions 
récentes à la loi Toubon surprennent également les francophones de Belgique : d'abord par l'ironie et les moqueries qu'elle rencontre en France, ensuite parce que cette loi qui vise essentiellement à protéger l'identité nationale française est réduite, dans l'esprit de la plupart des Français, à la dimension d'un contrôle tatillon sur la "pureté de la langue », par les « infractions » enfin en rapport avec un code du purisme qu'on reproche au ministre, sans que jamais ne soient évoquées les véritables dimensions de la question ${ }^{26}$.

De même, de nombreux francophones belges, surtout parmi les jeunes générations, ont cessé de revendiquer ou d'espérer pour leur langue, dans les organismes internationaux, une position équivalente à celle de l'anglais. Leur attitude sur ce plan n'est guère différente de celle qu'ils ont aussi sur le plan des relations extérieures où les cocoricos français les amusent ou les agacent. Par contre, beaucoup de francophones belges sont irrités par les comportements d'organismes ou de firmes françaises qui adoptent d'emblée l'usage de l'anglo-américain même lorsqu'on s'adresse à eux en français ou lorsque cet échange s'effectue entre francophones ${ }^{27}$. Cet empressement français à se soumettre à l'anglo-américain est d'autant plus mal supporté en Belgique qu'on constate qu'il s'accompagne, parfois chez les mêmes personnes, d'une incapacité à s'exprimer autrement qu'en termes hexagonaux et en référence à la situation strictement française, d'une difficulté, en d'autres termes, à s'ouvrir aux points de vue et aux sensibilités d'étrangers, fussent-ils francophones.

31 Les contradictions de la politique linguistique française, tant sur le plan intérieur envers les minorités allophones qu'à l'extérieur en poursuivant parfois une politique soit néocolonialiste, soit impérialiste, apparaissent moins aux francophones belges, sauf peutêtre à la très faible minorité des Wallons wallonants. Par contre, on semble assez sensible, du côté francophone, au rôle important que pourraient jouer la France et la langue française en tant que moteur de revendications contre l'américanisation des échanges, soit en s'associant à d'autres "grandes langues internationales ", telles que l'allemand et l'espagnol, soit en prenant la direction de revendications plus générales de la même manière que les revendications humanistes et libertaires de la Révolution française de 1789 auraient éclairé le monde.

Malgré les tensions nées de la coexistence de trois communautés sur son territoire, un pourcentage probablement élevé de Belges considère comme un bienfait personnel et collectif d'avoir dû s'adapter à une semblable diversité ethnique et linguistique. Du côté francophone, une majorité vraisemblablement croissante irait dans le sens de l'exigence d'une reconnaissance de sa propre identité et du développement d'une politique d'alliances entre les francophones et d'autres communautés linguistiques.

\section{Du côté des néerlandophones}

Le statut des néerlandophones de Belgique leur confère une originalité particulière dans la question des conséquences dans notre pays des politiques linguistiques de la France. Comme leurs compatriotes wallons dans leurs relations avec la France, les néerlandophones belges sont minoritaires et "provinciaux" par rapport aux néerlandophones des Pays-Bas dont ils parlent également une langue jugée parfois moins " pure ». À l'intérieur du pays, par contre, les néerlandophones belges représentent une majorité incontestable quel que soit d'ailleurs le mode de calcul des francophones. C'est eux également qui détiennent la plus grande partie du revenu national. Néanmoins, le souvenir des inégalités antérieures rejoint ici le sentiment d'appartenir à une 
communauté actuellement moins importante que la francophonie peut engendrer une méfiance, parfois mêlée de rancœur ou d'esprit de vengeance, envers les francophones de Belgique jugés (les Wallons surtout) par certains Flamands comme paresseux, pourris par le collectivisme, etc. Dans ce contexte, les sentiments d'ironie amusée ou d'agacement développés au sud à l'encontre de certains aspects de la politique française particulièrement en matière de langue sont amplifiés au nord et engendrent aisément la suspicion, sinon l'opposition.

Il y a donc lieu d'interpréter les positions, sans doute majoritaires, des néerlandophones belges comme favorables aux thèses françaises dans la mesure où la France développerait une politique globale d'alliances linguistiques, visant à renforcer les éléments identitaires des diverses composantes nationales sur les plans politique, économique, etc. Toutefois, mais sans doute ici les néerlandophones belges rejoignent-ils tous les laissés-pour-compte éventuels d'une semblable politique, ces alliés éventuels deviendraient-ils immédiatement des adversaires si la France devait, par exemple dans les discussions à venir sur la distinction entre langues officielles et langues de travail au sein de l'Union européenne, adopter une politique d'alliances qui négligerait le néerlandais. Ces adversaires se tourneraient alors vraisemblablement vers d'autres alliés potentiels et, à défaut, s'en remettraient au « tout à l'anglais » comme paraissent s'y résoudre, d'ailleurs apparemment sans état d'âme, leurs voisins néerlandais.

À cet égard, l'important effort fourni par la France et l'engouement certain, bien que très récent, que connaît dans ce pays l'enseignement du néerlandais devrait être davantage affirmé dans les Flandres que ce n'est le cas actuellement. Très sensibles sur ce point, les Flamands se montrent toujours très touchés lorsqu'on prend la peine de s'adresser à eux dans leur langue. Les firmes françaises qui désirent s'installer dans notre pays feraient beaucoup mieux, plutôt que de «simplifier » les relations en pratiquant l'unilinguisme anglais à destination des trois communautés linguistiques belges, d'exiger de leur personnel, français ou belge, la connaissance des langues des communautés dans lesquelles ces personnes sont appelées à pratiquer leur profession.

\section{NOTES}

1. Je tiens à remercier tout particulièrement Jean Klein, Jean-Marie Pierret et Maurice Van Overbeke qui m'ont fait l'amitié de relire ce texte et de formuler d'importantes remarques. Je porte cependant l'entière responsabilité de ce texte.

2. Les possibilités d'évolutions ultérieures deviennent minces puisqu'on approche progressivement des limites évolutives d'un droit constitutionnel qui doit, par définition, refuser l'abrogation des articles qui fondent l'État lui-même. Aussi, à part la fédéralisation éventuelle de nouvelles matières, telles que la sécurité sociale, la seule évolution future concevable serait la division du pays ou « séparatisme ». Sur tout ceci, voir aussi Beheydt Ludo et Jucquois Guy, « La Belgique. Trois langues, trois régions, trois communautés», dans Herreras José éd., 1992. Situations linguistiques dans les pays de la Communauté européenne, Lez Valenciennes, 1992, nº 14, 39-51. 
3. La communauté germanophone, forte d'environ 67000 personnes et située à l'est en bordure des frontières avec l'Allemagne, reste largement à l'écart des conflits et des tensions qui opposent et déchirent les communautés néerlandaise et française.

4. On appelle en général ce genre de solution un « compromis à la belge ».

5. La reconnaissance de la communauté germanophone se fit un peu plus tardivement de même que son ancrage définitif au sein de la région wallonne.

6. Actuellement, selon le budget établi pour l'année 1994, l'État fédéral dispose encore de $11 \%$ des revenus provenant des impôts, tandis que les régions et communautés ont vu leur budget s'accroître jusqu'à $32 \%$ du budget global de l'État.

7. Cf. supra.

8. Un certain nombre de francophones bruxellois hésitaient également sur ce point, mais en adoptant un point de vue opposé à celui des néerlandophones. Ils estimaient, en effet, que, tant que la Constitution n'aurait pas entériné définitivement (l'éventualité d'une révision ultérieure de celle-ci paraît effectivement très problématique) les frontières linguistiques de la future région bruxelloise, un espoir restait permis quant à l'élargissement de cette région aux communes périphériques où les francophones ne jouissaient que de «facilités » (cf. supra).

9. Le dominé (maintenant souvent le francophone) s'opposant, en quelque sorte, à la modification de son statut.

10. Ou celui des francophones dans d'autres communes bénéficiant du régime des "facilités ", comme ceux de la région des Fourons, etc.

11. Le volet linguistique du recensement périodique ayant été supprimé par les lois de 1962, il est devenu difficile, autrement que par des moyens indirects, d'évaluer le nombre de citoyens de chaque (sous-) communauté.

12. Les estimations les plus basses proviennent des milieux néerlandophones et les plus élevées des milieux francophones.

13. Le nombre d'unilingues wallons, s'il en subsiste, doit être extrêmement faible, de même d'ailleurs que le nombre des bilingues wallon-français. En l'absence de données statistiques, on en est réduit à des supputations sur le nombre des bilingues français-wallons qui ne devrait pas excéder - selon des estimations que me communique Jean-Marie Pierret - les $20 \%$ de la population, soit environ 600000 personnes. Le wallon est encore pratiqué par de nombreuses personnes au-dessus de cinquante ans et demeure fort vivace non seulement dans les campagnes (jusqu'à $65 \%$ de la population), mais aussi dans certaines grosses agglomérations industrielles (par ex. dans le Hainaut). À titre d'indication, le théâtre dialectal wallon a donné, en 1990, 1248 représentations devant un public de 264379 spectateurs payants. Par ailleurs, la Belgique connaît, comme tous les pays européens, un tassement démographique compensé par des flux migratoires en provenance anciennement des pays latins, puis de Grèce, ensuite du Maghreb et finalement de Turquie. Les immigrés sont plus nombreux en Wallonie et à Bruxelles qu'en Flandre et ils adoptent habituellement la langue majoritaire de la région d'accueil, le français au sud et à Bruxelles, le flamand au nord.

14. Pour être complet, il faudrait tenir compte également de la minorité francophone résidant au sein de la Communauté germanophone (de même d'ailleurs qu'il faut ajouter aux germanophones de cette dernière des minorités germanophones dans les communes wallonnes du canton de Malmedy et, au sud du pays, dans la région d'Arlon en lisière du Luxembourg).

15. Douze autres communes de la région flamande et treize de la région wallonne bénéficient du même régime à « facilité ».

16. Le fait que Bruxelles soit la capitale fédérale, mais ait également été choisie comme capitale de la communauté flamande, exacerbe les sensibilités des Bruxellois francophones. La situation est rendue encore plus complexe et confuse par la présence quotidienne de centaines de milliers de «naveteurs » - belgicisme que le Nouveau Petit Robert vient d'intégrer - provenant, pour les deux tiers environ, de la région flamande. Enfin, Bruxelles étant une des capitales de l'Union 
européenne et aussi le siège de grandes organisations internationales, la présence d'importantes et de nombreuses minorités allophones sur son territoire et dans sa périphérie accentue encore le caractère cosmopolite récent de cette ville très moyenne démographiquement.

17. Si on estime la population wallonne à un peu moins de 3200000 personnes, abstraction faite des 67000 germanophones, la population francophone de Bruxelles-Capitale à environ $80 \%$ de 964000 personnes, estimation moyenne ainsi qu'on l'a rappelé, soit à environ 770000 citoyens et celle des francophones de la périphérie à environ 450000 personnes (moyenne des estimations provenant des milieux francophones), on aboutit à un total de 4420000 personnes dont $72,4 \%$ pour la Wallonie et 27,6\% pour Bruxelles et sa périphérie. Toutefois, les francophones de la périphérie bruxelloise ne sont pas comptabilisés, pour la plupart des matières, avec les autres francophones, mais bien avec les néerlandophones. De plus, même dans les «facilités » qui leur sont accordées, ils restent dépendants d'un pouvoir de tutelle flamand. Par exemple, les enseignants francophones des écoles françaises de la périphérie sont désignés et payés par un pouvoir flamand.

18. Du côté de l'uniformité se retrouvent la volonté de contrôle et de centralisation (ou encore l'impérialisme et le colonialisme), du côté de la diversité, l'autonomie, la liberté et la capacité de décider à un niveau local.

19. Cf. par exemple le débat passionné concernant la mini-réforme orthographique intervenue facultativement ! - récemment.

20. Questionnement qui, malgré les apparences, n'a rien d'outrancier: songeons que nous développons des attitudes de colonisés, la langue n'étant pas davantage ici par rapport à la réalité complexe que le thermomètre par rapport à la maladie. $\mathrm{Cf}$. aussi infra.

21. Ces alliances resteront toutefois lettre morte si elles ne se concrétisent pas dans un ensemble de mesures sur le plan de l'enseignement des langues étrangères (ou mieux : "sur le plan de l'enseignement en langues étrangères »), de l'économie, du droit, etc.

22. La défense de la diversité civilisationnelle - linguistique et culturelle - est une question éthique et une question pragmatique. Les arguments et les alliances pour défendre l'un et l'autre points de vue ne sont évidemment pas les mêmes : ainsi, dans les organismes internationaux, il existe un intérêt au maintien d'une diversité des modes d'expression linguistique (le droit, l'économie, etc. ne sont pas les mêmes, conçus et exprimés dans une langue ou dans une autre, la variété est ici garantie de davantage de liberté pour tous). Le point de vue pragmatique ne permet cependant pas de faire droit à toutes les demandes de reconnaissance de toutes les communautés minoritaires, demandes que devrait rencontrer par contre le point de vue éthique. En effet, davantage de liberté pour tous ne peut compenser, pour les groupes minoritaires, la perte ou l'occultation d'éléments identitaires aussi importants que la langue.

23. Cf. supra Langues, régions et communautés.

24. Rappelons encore qu'on manque d'études, tant en France qu'en Belgique ou en d'autres pays francophones, sur les sensibilités à propos de ces questions. On en est donc réduit à une description « impressionniste » de la situation.

25. II suffira de rappeler l'importance de l'école louvaniste en citant les noms de grammairiens parmi lesquels Joseph Hanse ou André Goosse qui poursuit l'œuvre d'un autre grammairien belge, Maurice Grevisse. La relève est largement assurée et cela dans les deux filles de l'ancienne université de Louvain, établies à Louvain-la-Neuve (Brabant wallon) et à Leuven (Brabant flamand).

26. Revenant d'un bref séjour en Provence, nous avons eu l'occasion, à plusieurs reprises, de discuter de cela avec des chefs d'entreprise ou des cadres supérieurs qui, dans la même conversation, faisaient état qui d'un participe que le ministre aurait omis de mettre au féminin, qui d'un « euh.. » qui aurait émaillé tel de ses discours, etc. Plusieurs m'ont d'ailleurs montré les copies des lettres qu'ils avaient adressées à $\mathrm{M}$. Toubon pour le reprendre et lui rappeler les règles de notre langue. Aucun d'entre eux n'avait songé à lui faire part de la situation de dépendance 
dans laquelle ils étaient pourtant entrés, apparemment sans même s'en apercevoir, par exemple en recrutant et en engageant en anglais un collaborateur français destiné à travailler avec eux en France.

27. Pour illustrer le propos, voici deux exemples authentiques : une très grosse maison d'édition française publiant une revue spécialisée dans le domaine de la langue française adressait, il y a deux ou trois ans, ses factures d'abonnement entièrement rédigées en anglais et cela même à ses clients belges francophones. Dans une grosse université du nord de l'Espagne, le responsable du département de français nous a montré la lettre rédigée en français qu'il avait adressée à une importante firme d'informatique dont le siège est en France pour passer commande du matériel nécessaire pour équiper son département: la réponse lui parvint rédigée en anglais. Ajoutons deux exemples d'entreprises françaises installées en Belgique (notamment dans le domaine bancaire et dans celui de l'informatique), où nous avons fait une expérience semblable: un courrier en français appelle une réponse en anglais! Certains francophones belges se résolvent d'ailleurs à adopter une même attitude lorsqu'on leur fait valoir que ce serait la meilleure manière d'éviter ou d'apaiser les conflits linguistiques entre néerlandophones et francophones belges. C'est ainsi qu'au Cercle belge de linguistique on a contraint des francophones, spécialistes du français, en réunion à Liège, à s'exprimer entre eux en anglais... On a fait savoir récemment qu'on avait suspendu ce "règlement linguistique ». Quel souffle de liberté ! Cf. aussi la note précédente.

\section{RÉSUMÉS}

Après une analyse de l'évolution historique et sociolinguistique des communautés francophone et néerlandophone de Belgique, l'auteur examine les répercussions dans ce pays de la politique linguistique menée en France tant en ce qui concerne la langue française que les langues régionales.

INDEX

Index géographique : France, Belgique

Mots-clés : politique linguistique, langue régionale, langue de minorité, statut linguistique

\section{AUTEUR}

GUY JUCQUOIS

Université de Louvain 